\title{
Influence of a roughness length error on vertical wind speed extrapolation for 2D ideal hills using an OpenFOAM ${ }^{\circledR}$ RANS simulation
}

\author{
Hanan Einav-Levy, Aviv Rosen ${ }^{\mathrm{a}}$, and Reuven Shenkar ${ }^{\mathrm{b}}$ \\ Technion - Israel Institute of Technology, Faculty of Aerospace Engineering, 32000 Haifa, Israel
}

\begin{abstract}
In modelling wind flow over a flat and complex terrain, the choice of roughnesslength-distribution is critical for accurate wind speed predications. This choice is often made based on the Davenport scale [1]. A satellite or aerial image of the location is used [2-4] and subjective or objective measures are used for translating the image into roughness length - $z_{0}$ - based on the Davenport scale. The choice of a Davenport table value is generally regarded as within an error of plus or minus one table value, leading to a $\pm 6 \%$ error in wind speed predictions for a flat terrain in neutral conditions [5]. In the paper this error is studied for a non-flat terrain, using a series of CFD simulations for 2D hills of various combinations of aspect ratio and steepness. Results show that the wind speed prediction error as a result of a "wrong" parameterization decreases with hill steepness until separation occurs. As a result of separation the error increases slightly, and then decreases again with a further increase in hill steepness.
\end{abstract}

\section{Introduction}

Vegetation and curvature affect the amplitude and vertical shear profile of wind velocity and turbulence in the atmospheric boundary layer close to the ground [6]. Such changes can increase or decrease the performance of wind turbines by enhancing or reducing the wind power availability. When modeling the wind flow over any terrain, from gentle hills to a complex mountainous terrain, the vegetation cover is normally classified according to the roughness length. For neutral atmosphere conditions, which are commonly assumed in wind flow simulations for wind turbine micro-siting, the roughness length influences the vertical shear of the horizontal wind speed in flat terrain through the following logarithmic law expression:

$$
U(z)=\frac{u_{*}}{k} \ln \left(\frac{z}{z_{0}}\right)
$$

$U(z)$ is the wind speed at a height $z, u_{*}$ is the friction velocity and $k$ is the von Karman constant (typically taken as 0.4).

\footnotetext{
ae-mail: rosen@aerodyne.technion.ac.il

be-mail: rshenkar@tx.technion.ac.il
}

This is an Open Access article distributed under the terms of the Creative Commons Attribution License 2.0, which permits unrestricted use, distribution, and reproduction in any medium, provided the original work is properly cited. 


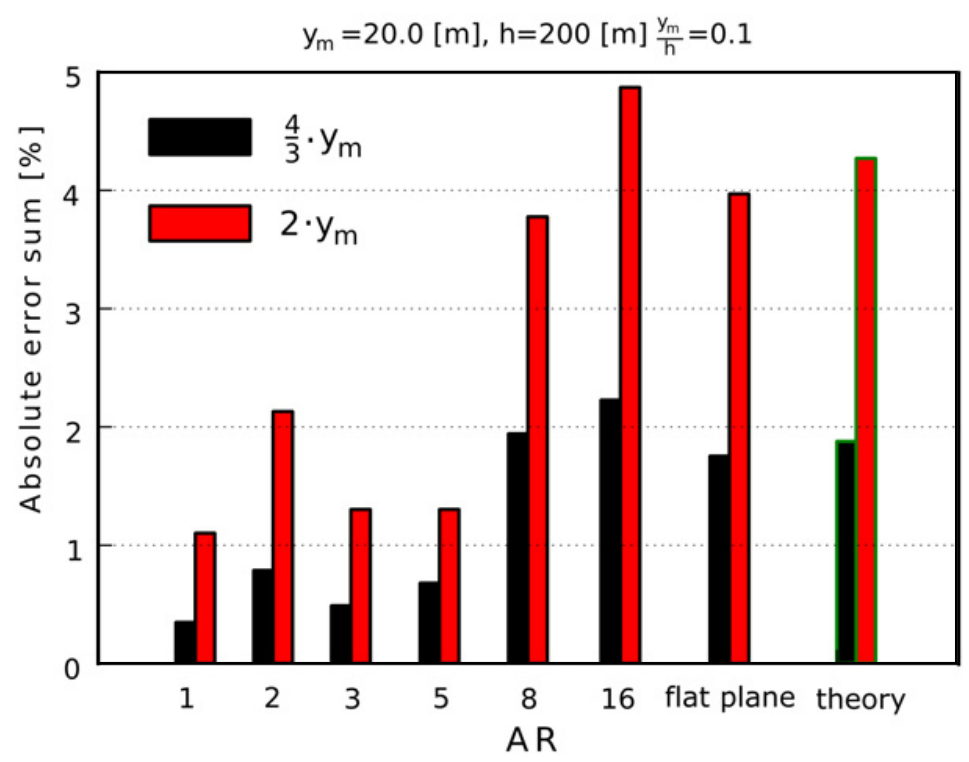

Figure 1. Error in vertical wind speed extrapolation for different hill $A R$ 's, as a result of roughness length classification error.

\section{Influence of roughness length estimation on simulated wind speed}

Using the above assumption and the Davenport scale [1], a typical error as a result of a wrong roughness height classification (one table value lower or higher Davenport scale) will create a $\pm 6 \%$ error in wind speed predictions [5]. For hills or a complex terrain, the error is expected to be reduced as the hill influence will drive the flow acceleration along-side the terrain roughness, and thus will reduce the influence of the roughness length error on the overall wind acceleration. The authors are not aware of a previous systematic investigation of this error; the present research is aimed at quantifying this error for different terrains by using state of the art CFD simulations.

\section{Methods}

The simulations are performed for 2D hills defined by the following equation [7]:

$$
\frac{y(x)}{H}=-\frac{1}{6.04844}\left[J_{0}(\Lambda) I_{0}\left(\Lambda \frac{x}{a}\right)-I_{0}(\Lambda) J_{0}\left(\Lambda \frac{x}{a}\right)\right] .
$$

The last equation applies for $|x| \leq a$, while $y$ equals zero elsewhere. $\Lambda=3.1926$, while $J_{0}, I_{0}$ are a Bessel function of the first kind and a modified Bessel function of the first kind, respectively. $H=200$ $[\mathrm{m}]$ is the hill height and $a$ is the hill half-length. The hill aspect ratio $(A R)$ is defined as $A R=a / H$. Simulations are performed for various $A R$ values $(1,2,3,5,8,16)$ and a flat terrain.

OpenFOAM 2.1.1 is used on an 8 core PC with a 12 Gb memory.

Solver: The simpleFoam steady state RANS solver is used, with the $K-\varepsilon$ turbulence closure model.

Mesh: The mesh is created with the OpenFOAM blockMesh utility, using three blocks - upwind, hill centered and downwind. Domain height is 3000 [m], upwind domain length is set according to the hill 
length as $5 a$ and downwind domain length is $10 a$. The first cell height is varied according to roughness, to present a height of 2-20 times the roughness length [8]. Typical cell count is 50,000-100,000.

Boundary conditions: The inlet and top of the domain velocity are set to atmBoundaryLayerInletVelocity (Eq. (1)). A no-slip condition is set on the ground, and zero gradient at the outlet. The pressure is set to zero gradient at all boundaries, and in the outlet it is fixed to 0 . Nut $\left(v_{t}\right)$ is set to nutRoughWallFunction at the ground and "calculated" at all other boundaries. Epsilon is set in the inlet and top according to the height decreasing function atmBoundaryLayerInletEpsilon, epsilonWallFunction at the ground and zero gradient at the outlet. The turbulent kinetic energy $K$ is constant at the inlet and top boundaries, $k q R$ WallFunction at the ground and zero gradient at the outlet. Values for inlet parameters are chosen according to Richards and Hoxey [9].

Simulation analysis: The results are analyzed according to the assumption that an anemometer is placed at a height $y_{m}$ above the hill top, and the simulation is used to predict the speed at 100\% and 50\% higher points. Different scenarios are tested, for typical hub-heights to hill-height ratios. An example for $y_{m}=20[\mathrm{~m}]$ is presented below.

\section{Results}

The flat terrain simulation is similar to the theoretical wind speed error due to error propagation from roughness length error $(\sim 4 \%)$. There is a small increase for low hills $(\sim 5 \%$ for $A R=16)$ while an increase in hill steepness leads to a reduction in the error. The onset of separation $(1.3 \%$ for $A R=5)$ results in a slight increase in the error $(2 \%$ at $A R=2)$, while a further increase in steepness results in a reduction of the error.

\section{Conclusions}

The CFD simulations present the influence of a decrease in roughness length on the error in wind speed estimation for various 2D hill aspect ratios. Recently a new proposal for the Davenport scale was presented, based the desire to have equal wind speed errors as a result of an increase or a decrease in the assumed roughness height [10]. Since the extrapolated wind speed error for positive or negative error is influenced by the terrain complexity, such a proposal will have to take this into account by using simulations like the ones presented here (a 2D hill can be seen as representing wind acceleration over a long ridge) and for typical 3D scenarios. In the future this investigation will be extended to include 3D hills and a terrain of inhomogeneous roughness.

\section{References}

[1] J. Wieringa, 3rd European and African Conference on Wind Engineering, Eindhoven, The Netherlands (2001)

[2] J. Colin, R. Faivre, M. Menenti, Hydrol. Earth Syst. Sci. Discuss. 7, pp. 3397-3421 (2010)

[3] C. Hasager, N. Nielsen, N. Jensen, E. Boegh, J. Christensen, E. Dellwik, H. Soegaard, BoundaryLayer Meteorology 109, pp. 227-254 (2003)

[4] N. I. Ramli, M. I. Ali, M. S. Saad, T. A. Majid, The Seventh Asia-Pacific Conference on Wind Engineering, Taipei, Taiwan (2009)

[5] J. Wieringa, Quarterly Journal of the Royal Meteorological Society 112, pp. 867-889 (1986)

[6] E. Petersen, N. Mortensen, L. Landberg, J. Højstrup, H. Frank, Wind Energy 1, pp. 25-45 (1998) 


\section{ITM Web of Conferences}

[7] J. Harju, H. Nilsson, E. Furbo, Evaluation of turbulence models for prediction of flow separation at a smooth surface, Uppsala Universitet report (2009)

[8] B. Martinez, Wind resource in complex terrain with OpenFOAM, Risø-DTU report (2011)

[9] P. Richards, R. Hoxey, Journal of Wind Engineering and Industrial Aerodynamics 46-47, pp. 145-153 (1993)

[10] E. C. C. Choi, The Seventh Asia-Pacific Conference on Wind Engineering, Taipei, Taiwan (2009) 